\title{
Identification of Nocardiopsis dassonvillei in a Blood Sample from a Child
}

\author{
${ }^{1}$ Flavio Lejbkowicz, ${ }^{1}$ Raya Kudinsky, ${ }^{1}$ Luisa Samet, ${ }^{1}$ Larissa Belavsky \\ ${ }^{1}$ Miriam Barzilai and ${ }^{2}$ Svetlana Predescu \\ ${ }^{1}$ Clinical Microbiology Laboratory, Western Galilee Hospital, Naharyia \\ ${ }^{2}$ Microbiology Laboratory, Rambam Medical Center, Haifa, Israel
}

\begin{abstract}
Nocardiopsis dassonvillei is an environmental aerobic actinomycete producing a funguslike mycelium and aerial hyphae. Here we report the first Nocardiopsis dassonvillei isolated from a blood sample from a 3-year-old child hospitalized with fever, respiratory difficulty and cough. To the best of our knowledge this is the first time this organism has been detected in the BacT/Alert system. This Nocardiopsis was designated Nocardiopsis dassonvillei based on morphological and physiological tests.
\end{abstract}

Key words: Nocardiopsis, Blood Sample

\section{INTRODUCTION}

In 1976 Meyer described the Nocardiopsis genus [1]. This new genus was characterized according to its mode of sporulation, molecular genetic studies, numerical taxonomic and chemotaxonomic analysis [16]. Nocardiopsis genus is an aerobic actinomycete that includes several species [2, 5-8]. Nocardiopsis dassonvillei is one of the species and it was originally named Streptothrix dassonvillei, changed to Nocardia dassonvillei, or Actinomadura dassonvillei and finally Nocardiopsis dassonvillei. This genus resembles fungi and has been isolated from soil, water and organic matter. Not many members of the Nocardiopsis genus have been involved with human pathogenesis. Nocardiopsis dassonvillei is to a certain extent an exception and few reports describe its involvement in human pathogenesis. It has been implicated in a case of cellulitis of the arm [9] and in cases of alveolitis or suppurative pulmonary infection [10, 11]. Here we report the first Nocardiopsis dassonvillei isolated from a blood sample from a 3-year-old child hospitalized with fever and respiratory difficulty. This uncommonly pathogen was recovered in a pediatric blood culture bottle in the Bact/Alert system (Organon Teknika). To the best of our knowledge this is the first time this organism has been detected in the BacT/Alert system and in a blood sample from a child.

\section{CASE REPORT}

A 3-year-old child was hospitalized with fever and some signs of respiratory distress with a productive cough. A blood sample was sent to the laboratory and bacterial growth was detected after 5 days in the BacT/Alert system (Organon Teknika). Microscopic analysis demonstrated a gram-positive filamentous bacterium with long hyphae and acid-fast negative. The only isolate was subcultured on nonselective media, including blood, chocolate and Sabouraud's dextrose agar. Macroscopic analysis showed very dry and dissimilar crinkled colonies, yellowish to brown pigmentation and a soil-like odor. After further incubation, part of the colonies turned white. The aerial hyphae were long and branched with a zigzag aspect. The sporulation was complete and fragmented into spore chains of various sizes.

There is not a guideline for susceptibility tests for Nocardiopsis dassonvillei. Primarily, the disk diffusion technique in both plates Mueller Hinton agar and Muller Hinton agar supplemented with blood was used for susceptibility tests for a broad range of antibiotics and later the Etest strip (a quantitative susceptibility test, trademark of AB Biodisk, Solna, Sweden) was used to establish the minimal inhibitory concentration for trimethoprim and gentamicin. The organism showed complete susceptibility on Etest strips to trimethoprim and aminoglycosides, but total resistance to cephalosporins and quinolones. Initially, an empirical therapy for bacterial infection was started with erythromycin. This course of therapy was changed after bacterial growth and susceptibilities were determined. Accordingly, trimethoprim and gentamicin were added to his antimicrobial regimen for 2 weeks with subsequent improvement in his clinical status. The treatment was kept under submaximal dosage for another 3 months.

Several tests were performed to characterize this organism. The bacterium tested positive for urease, gelatin hydrolysis and sensitive to lysozyme. Inoculation on adenine, casein, tyrosine, xanthine and hypoxanthine media resulted in hydrolysis. Acid formation from different carbohydrates and other characteristics tested are summarized in Table 1-3 in comparison to closed-related organisms.

\section{DISCUSSION}

Nocardiopsis dassonvillei is an environmental aerobic actinomycete and its medical importance was initially 
Table 1: General Characteristics

\begin{tabular}{|c|c|c|c|c|}
\hline Organism & Cell-wall type & $\begin{array}{l}\text { Cell-wall lipid } \\
\text { Nocardo-mycolic acid }\end{array}$ & Acid-fast & Lysozyme \\
\hline Nocardiopsis dassonvillei & $\begin{array}{l}\text { III/C; meso-DAP, } \\
\text { No characteristic sugar }\end{array}$ & Absent & $(-)$ & Susceptible \\
\hline Nocardia & $\begin{array}{l}\text { IV; meso-DAP, } \\
\text { Arabinose, galactose }\end{array}$ & Present & $(+)$ & Resistant \\
\hline Actinomadura & $\begin{array}{l}\text { III/B; meso-DAP, } \\
\text { Madurose }\end{array}$ & Absent & $(-)$ & Susceptible \\
\hline Streptomyces somaliensis & $\begin{array}{l}\text { I; 11-DAP, glycine, } \\
\text { No characteristic sugar }\end{array}$ & Absent & $(-)$ & $\begin{array}{l}\text { Susceptible } \\
\text { (Variable) }\end{array}$ \\
\hline
\end{tabular}

Table 2: Decomposition of Media

\begin{tabular}{|c|c|c|c|c|c|c|c|}
\hline Organism & Adenine & Casein & Tyrosine & Xanthine & Hypoxanthine & Gelatin & Urea \\
\hline Nocardiopsis alba $^{(\mathrm{A})}$ & $(+)$ & $(+)$ & $(+)$ & $(+)$ & $(+)$ & (ND) & $(+)$ \\
\hline Nocardiopsis dassonvillei & $(+)$ & $(+)$ & $(+)$ & $(+)$ & $(+)$ & $(+)$ & $(+)$ \\
\hline Nocardiopsis & $(+)$ & $(+)$ & $(+)$ & $(+)$ & $(+)$ & (ND) & $(+)$ \\
\hline Synnemataformans ${ }^{(\mathrm{A})}$ & & & & & & & \\
\hline Nocardia brasiliensis & $(+)$ & $(+)$ & $(+)$ & $(-)$ & $(+)$ & $(+)$ & $(+)$ \\
\hline Actinomadura madurae & (ND) & $(+)$ & $(+)$ & $(-)$ & $(+)$ & $(+)$ & $(-)$ \\
\hline Streptomyces somaliensis & (ND) & $(+)$ & $(+)$ & $(-)$ & $(-)$ & $(+)$ & $(-)$ \\
\hline
\end{tabular}

Table 3: Acid Formation from

\begin{tabular}{|c|c|c|c|c|c|c|c|}
\hline Organism & Cellobiose & Xylose & Arabinose & Inositol & Maltose & Mannitol & Lactose \\
\hline Nocardiopsis alba $^{(\mathrm{A})}$ & $(+)$ & $(-)$ & (ND) & $(-)$ & $(+)$ & $(-)$ & (ND) \\
\hline Nocardiopsis dassonvillei & $(+)$ & $(+)$ & $(+)$ & $(-)$ & $(-)$ & $(+)$ & $(-)$ \\
\hline Nocardiopsis & $(+)$ & $(+)$ & (ND) & $(+)$ & $(+)$ & $(+)$ & (ND) \\
\hline Synnemataformans ${ }^{(\mathrm{A})}$ & & & & & & & \\
\hline Nocardia brasiliensis & $(-)$ & $(-)$ & $(-)$ & $(+)$ & (ND) & $(+)$ & $(-)$ \\
\hline Actinomadura madurae & $(+)$ & $(+)$ & $(+)$ & $(+/-)$ & (ND & $(+)$ & $(+)$ \\
\hline Streptomyces somaliensis & $(-)$ & $(-)$ & $(-)$ & $(-)$ & (ND) & $(-)$ & $(-)$ \\
\hline
\end{tabular}

$(-)=$ Negative $\quad(+)=$ Positive $\quad \mathrm{ND}=$ Not done

$(\mathrm{A})=$ Data compiled from literature

related to mycetoma $[12,13]$. In addition, it has been involved in cutaneous and pulmonary infections [9-11]. This organism may cause some difficulties in identification because of its close characteristics to other organisms. Other reports have identified Nocardiopsis dassonvillei on the basis of its cell wall fatty acid analysis and 16S rRNA gene sequence [2, 5$8,14-16]$, but these methods are not generally available in the routine laboratory. Here, the Nocardiopsis was designated Nocardiopsis dassonvillei using morphological and several physiological tests.

Previous publication has reported the first Nocardiopsis dassonvillei in a blood isolate from a 60-year-old man presented with cholangitis [16]. The organism was detected using the BACTEC aerobic bottle in the BACTEC automated instrument. Our isolate was the first pediatric isolate retrieved from a blood sample. In addition, to the best of our knowledge this is the first time this organism has been detected in the BacT/Alert system. Apparently, this organism is not a common contaminant of blood samples and not a saprophytic organism in the human body. Since there was no other medical evidence to explain the illness and no other etiological agent was isolated, we have attributed the illness condition to this organism.

Nocardiopsis dassonvillei is part of the dust-borne bacteria in schools, children's day-care centres and especially in animal sheds $[17,18]$. The present child lives in a rural village, in an environment with precarious hygienic conditions; thus, the possibility that this organism was contracted from the soil is likely to be reasonable. On the other hand a less probable, but still possible way of contraction of the spores is through a familial member working in an animal shed. Precarious hygiene conditions may be predisposing factors to acquire infection. Furthermore, as discussed in Andersson et al., [17], endotoxin from gram-negative bacteria and cell wall components of actinomycetes may be the causative agent for farmers' lung [18]. This is possible when assuming that inhalation of the bacteria from the soil is sufficient to induce hypersensitivity and pneumonitis. The hypersensitivity condition favors the establishment of the bacteria that can be propagated to the bloodstream leading to bacteremia. 
Chocolate agar/ Blood agar

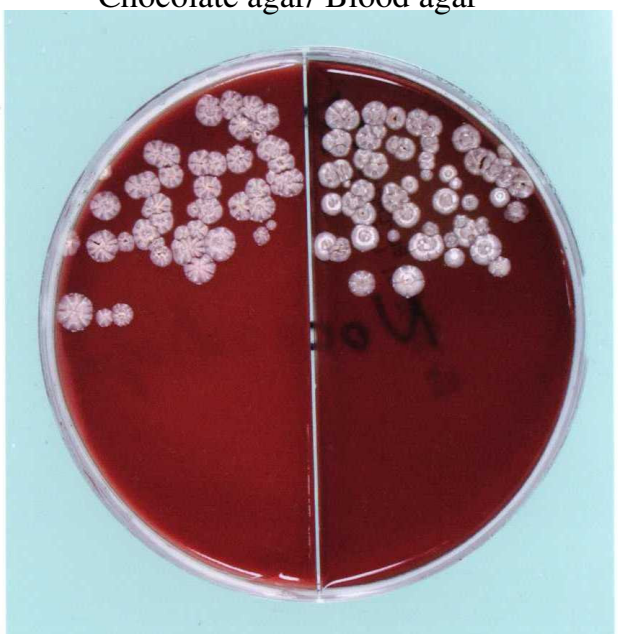

Gelatin Hydrolysis

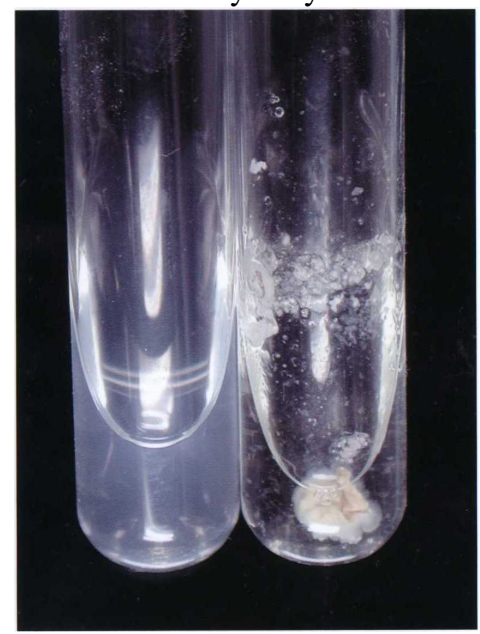

Casein Hydrolysis

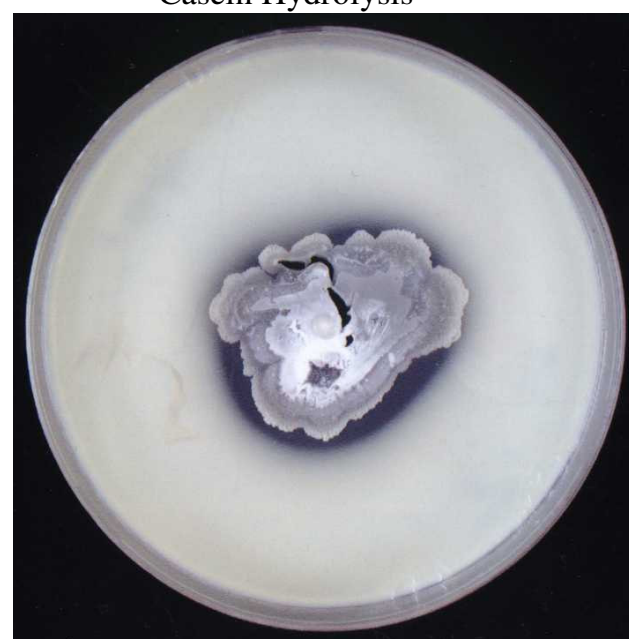

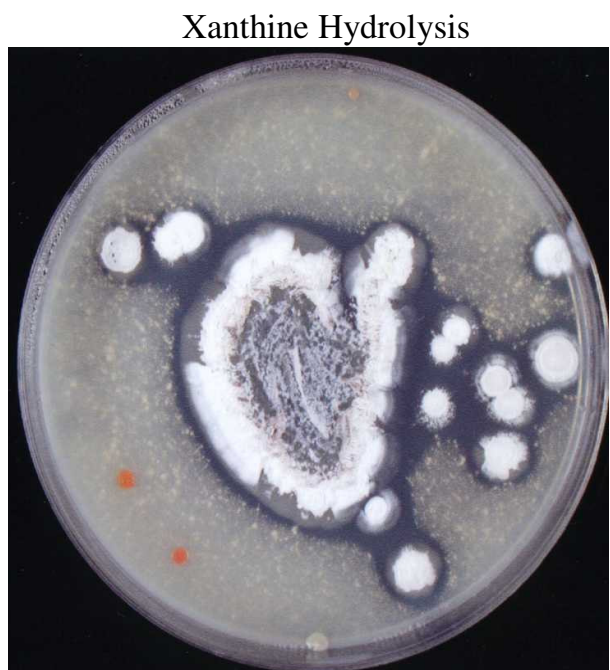

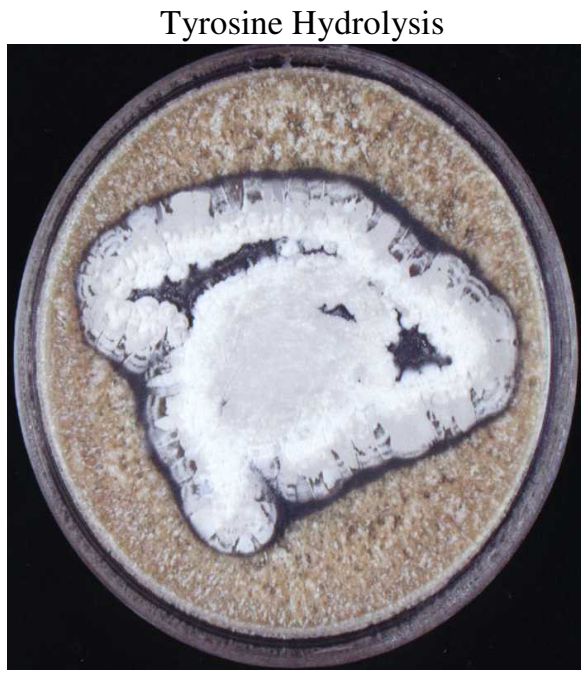

Fig. 1: Macroscopic Appearance of Nocardiopsis dassonvillei and Hydrolysis of Different Substrates

Nocardiopsis dassonvillei is not a human pathogen commonly retrieved from a blood sample and most of the routine laboratories are not familiar with aerobic actinomycetes. Furthermore, identification of this organism in a routine clinical laboratory is rare, since it cannot be identified by regular routine tests and a series of extra tests should be performed. In the future when molecular techniques will be accessible to diagnostic laboratories, the differentiation between this and other closely related organisms should shed light on a wide range of medically important pathogens by leading to a more detailed understanding of relations between pathogen and illness. 


\section{ACKNOWLEDGEMENTS}

The authors thank Dr. Izabella Lejbkowicz and Dr. Anne Kirshenbaum for their critical reading.

\section{REFERENCES}

1. Meyer, J., 1976. Nocardiopsis a new genus of the order actinomycetales. Intl. J. Syst. Bacteriol., 26: 487-493.

2. Fischer, A., R.M. Kroppenstedt and E. Stackebrandt, 1983. Molecular-genetic and chemotaxonomic studies on Actinomadura and Nocardiopsis. J. Gen. Microbiol., 129: 34333446.

3. Athalye, M., M. Goodfellow, J. Lacey and R.P. White, 1985. Numerical classification of Actinomadura and Nocardiopsis. Intl. J. Syst. Bacteriol., 35: 86-98.

4. Grund, E. and R.M. Kroppenstedt, 1990. Chemotaxonomy and numerical taxonomy of the genus Nocardiopsis Meyer 1976. Intl. J. Syst. Bacteriol., 40: 5-11.

5. Rainey, F.A., N. Ward-Rainey, R.M. Kroppenstedt and E. Stackebrandt, 1996. The genus Nocardiopsis represents a phylogenetically coherent taxon and a distinct actinomycete lineage: Proposal of Nocardiopsaceae fam. nov. Intl. J. Syst. Bacteriol., 46: 1088-1092.

6. Yassin, A.F., F.A. Rainey, J. Burghardt, D. Gierth, J. Ungerechts, I. Lux, P. Seifert, C. Bal and K.P. Schaal, 1997. Description of Nocardiopsis synnemataformans sp. nov., elevation of Nocardiopsis alba subsp. prasina to Nocardiopsis prasina comb. nov. and designation of Nocardiopsis antarctica and Nocardiopsis alborubida as later subjective synonyms of Nocardiopsis dassonvillei. Intl. J. Syst. Bacteriol. 47: 983-988.

7. Tul'skaya, E.M., G.M. Streshinskaya, I.B. Naumova, A.S. Shashkov and L.P. Terekhova, 1993. A new structural type of teichoic acid and some chemotaxonomic criteria of two species Nocardiopsis dassonvillei and Nocardiopsis antarcticus. Arch. Microbiol., 160: 299-305.

8. McNeil, M.M. and J.M. Brown, 1994. The medically important aerobic actinomycetes: Epidemiology and microbiology. Clin. Microbiol. Rev., 7: 358-417.
9. Philip, A. and G.D. Roberts, 1984. Nocardiopsis dassonvillei cellulitis of the arm. Clin. Microbiol. Newsl., 6: 14-15.

10. Bernatchez, H. and E. Lebreux, 1991. Nocardiopsis dassonvillei recovered from a lung biopsy and a possible cause of extrinsic alveolitis. Clin. Microbiol. Newsl., 6: 47-55.

11. Mordarska, H., J. Zakrzewska-Czerwinska, M. Pasciak, B. Szponar and S. Rowinski, 1998. Rare, suppurative pulmonary infection caused by Nocardiopsis dassonvillei recognized by glycolipid markers. FEMS Immunol. Med. Microbiol., 21: 47-55.

12. Sindhuphak, W., E. Macdonald and E. Head, 1985. Actinomycetoma caused by Nocardiopsis dassonvillei. Arch. Dermatol., 121: 1332-1334.

13. Ajello, L., J. Brown, E. Macdonald and E. Head, 1987. Actinomycetoma caused by Nocardiopsis dassonvillei. Arch. Dermatol., 123: 426.

14. Naumova, I.B., A.S. Shashkov, E.M. Tul'skaya, G.M. Streshinskaya, Y.I. Kozlova, N.V. Potekhina, L.I. Evtushenko and E. Stackebrandt, 2001. Cell wall teichoic acids: Structural diversity, species specificity in the genus Nocardiopsis and chemotaxonomic perspective. FEMS Microbiol. Rev., 25: 269284.

15. Weisburg, W.G., S.M. Barns, D.A. Pelletier and D.J. Lane, 1991. 16S ribosomal DNA amplification for phylogenic study. J. Bacteriol., 173: 697-703.

16. Beau, F., C. Bollet, T. Coton, E. Garnotel and M. Drancourt, 1999. Molecular identification of a Nocardiopsis dassonvillei blood isolate. J. Clin. Microbiol., 37: 3366-3368.

17. Andersson, A.M., N. Weiss, F. Rainey and M.S. Salkinoja-Salonen, 1999. Dust-borne bacteria in animal sheds, schools and children's day care centres. J. Appl. Microbiol., 86: 622-634.

18. Peltola, J.S., M.A. Andersson, P. Kampfer, G. Auling, R.M. Kroppenstedt, H.J. Busse, M.S. Salkinoja-Salonen and F.A. Rainey, 2001. Isolation of toxigenic Nocardiopsis strains from indoor environments and description of two new Nocardiopsis Species, N. exhalans sp. nov. and N. umidischolae sp. nov. Appl. Environ. Microbiol., 7: 4293-4304. 